\title{
The Next Big Thing is Really Big!! Magnification in Dentistry
}

\author{
Shweta Kishor Sedani ${ }^{1}$, Anuja Dhananjay Ikhar², Akshay Pramod Thote ${ }^{3}$ \\ 1, 2 Department of Conservative Dentistry and Endodontics, Sharad Pawar Dental College, Datta \\ Meghe Institute of Medical Sciences, Maharashtra, India. ${ }^{3}$ Department of Endodontics, Sharad \\ Pawar Dental College, Datta Meghe Institute of Medical Sciences, Maharashtra, India.
}

\section{ABSTRACT}

\section{BACKGROUND}

The advances in the magnification sector have revolutionised the endodontic treatment from tactile and radiograph based to the vision driven. Using magnification tools like loupes, microscopes, rod lens endoscopes and oroscopes in the endodontic practice enables the clinician to magnify the field of treatment further than that by naked eyes. Suitable illumination along with magnification adds to the success of any dental procedure. Dating back to 1870, surgical loupes have been used. Apotheker and Jako introduced microscope in dentistry for the first time in 1978. Carr, in 1992 highlighted the role of the surgical operating microscope in endodontics. Various applications of surgical operating microscopes include minimally invasive surgical techniques like in periodontal regenerative procedures, adhesive dentistry and various procedures in endodontics like caries detection, for hidden canals, pulp stone removal, instrument removal, surgical endodontics, retreatment, perforation seal etc. Parts of operating microscope include optical components, objective lens, binocular tubes, eyepieces, lighting unit and mounting system. Accessories include motorised / foot-controlled adjustment of focal length, mechanical optical rotating assembly (MORA) interface and beam splitter.

Magnification aids assist in producing higher quality procedures due to better precision and accuracy. Using the microscope aids improved ergonomics for the operator. Using loupes or microscopes improves the clarity in treatment plan as well as its execution. The magnification aids with camera and video monitor attached, enhance the patient education and better documentation. A strong consideration should be given to adopt using the concept of magnification. This review intended to explain the use of these tools in dental procedures for better accuracy, handling and thoroughness, which will lead to fewer procedural errors.

\section{KEY WORDS}

Magnification, Illumination, Loupes
Corresponding Author: Dr. Shweta Kishore Sedani, 202, Gokul Park 1, Near Kachore Lawns, Beesa Belatarodi Road, Manish Nagar, Nagpur - 440005, Maharashtra, India. E-mail:doc.shwets@gmail.com

DOI: $10.14260 / j e m d s / 2021 / 231$

How to Cite This Article: Sedani SK, Ikhar AD, Thote AP. The next big thing is really big!! magnification in dentistry. I Evolution Med Dent Sci 2021;10(15):1083-1087, DOI: 10.14260/jemds/2021/231

Submission 24-04-2020,

Peer Review 09-02-2021,

Acceptance 15-02-2021,

Published 12-04-2021.

Copyright (C) 2021 Shweta Kishor Sedani et al. This is an open access article distributed under Creative Commons Attribution License [Attribution 4.0 International (CC $B Y$ 4.0)] 


\section{BACKGROUND}

Perception of conventional endodontic treatment and surgical treatment has been restricted to the two-dimensional dental radiograph which is representative of a three dimensional biological system. Achieving the hermetic seal after debridement of canal space is the basic for the success of endodontic therapy. Proper illumination and adequate magnification adds to the endodontic success. The incorporation of magnification tools like loupes, microscopes and endoscopes in the endodontic practice facilitates the clinician to magnify a particular field of treatment further than that of naked eye.

The idea of using magnification for microsurgery was presented into medicine in the course of late 19th century. Binocular microscope for surgeries of ear was first used by Carl Nylen in 1921 who was considered as the father of microsurgery. In 1978 Apotheker and Jako introduced microscope in dentistry for the first time. This led to a gradual turn of event in 1992 where Carr, prepared and issued a published work that described \& highlighted function of surgical operating microscopic device in endodontic surgeries.1-6

It is well proven that magnification gives better and clear vision.

A magnified image with enhanced clarity provides a detailed description of an object than numerous words put in collaboration. So it can be considered as an eye opener to dental clinical practice. In 1979, Daniel defined microsurgery in broad terms. Microsurgery includes three different principles $^{2}$ -

1. Upgrading motor skills, thus enhancing surgical ability.

2. Highlight passive closing of wound with cautious apposing of primary wound edge.

3. Using microsurgical armamentarium and suturing to lessen tissue trauma.

According to Kim et al. the "Microsurgical Triad" consists of magnification, illumination and instruments. This review intended to explain the use of these tools in dental procedures for better accuracy, handling and thoroughness, while having fewer procedural errors.

\section{TYPES AND PRINCIPLES OF MAGNIFICATION SYSTEMS}

Magnification enhanced dentistry includes the use of 2 types of visual magnification tools that include loupes and dental or surgical operating microscope (DOM).

\section{Loupes}

Loupes are the most commonly used magnification systems in dentistry. It has two monocular microscopes, having holder to shoulder lenses. These lenses are angled which are focused on an object to form enlarged images. Loupes have convergent lenses. Magnification ranges from $\times 1.5$ to $\times 10$. It is better to start with loupes for magnification, which helps the operator to adjust the eye. The most common disadvantage of using loupes is there is convergence of eyes while perceiving an image (Keplerian optics), that may lead to draining of eyes due to fatigue. Extended use of ill fitted loupes may even cause the vision changes. ${ }^{7}$

Three types of loupes are put to use in daily practice

\section{Simple Loupes}

It involves a couple of solitary, side-by-side meniscus lenses with dual surfaces that refract. Through the first surface there is entry of light whereas through the second surface there is exit of light. It is economical. Drawback is that it has partial capabilities. They also come across spherical \& chromatic anomalies that lead to image distortion of the object.

\section{Compound / Telescopic Loupes}

Telescopic loupes comprise of several lenses with fundamental air spaces, thus letting adjustments in working distance, magnification \& depth of field without escalating the bulk or dimension.

\section{Prism Loupes}

These are more advanced containing Pechan / Schmidt prisms which extend light pathway via one sequence of mirror images. This is done by virtual folding of light to reduce loupe barrel. These improve magnifying factor, deliver greater fields of view (FOV), broader depths of field, \& longer WDs.8,9 This aspect must be sought by dental professionals while they select a magnifying loupe.

\section{Surgical operating Microscope}

Galilean principles are used to design the surgical operating microscopes in dentistry. They integrate the habit of magnification loupes along with a binocular viewing system and a magnification changer. It employs the use of a parallel binoculars that protect against the eye fatigue and strain. Fully coated optics and achromatic lenses having good contrast vision and high resolution are also included. They use coaxial fiber optic illumination that provides with a bright and adjustable light spot which is circle in shape. This is parallel to visual viewing axis. The light does not contain shadow which enables accurate viewing of pathologies, its recording in motion, and its document making and managing of all dental \& surgical course of action. ${ }^{2}$ Beam splitter provides visualisation of magnified images on monitor screen for patient as well as assistant.

Surgical operating microscope has a lens system which enables excellent illumination of working area along with the stereoscopic vision at the magnification of nearly $\times 4-40$. Light beam falls along the line into observer's retina thus eliminating the convergence and straining of eye muscle, mainly lateral rectus. The different microscopic parts are 9-11

\section{Optical Components}

Magnification (Galilean) changer contains a cylinder, in which 2 telescope systems are built having varied magnification factors. A total of 4 different magnification levels exist. Magnification factor determines the power of magnification.

\section{Objective Lens}

The image is presented by single objective once processed by magnification changer. This projects light from source twice into the operation area by the prisms for deflection. The most widely used objective in dentistry has been $250-300 \mathrm{~mm}$. 


\section{Binocular Tubes}

These are of straight / inclined types. Inclined tubes give better adapting view. Because the operator is able to modify the tubes without altering his posture, (back, head or neck) they improve the feasibility for enhancing ergonomics.

\section{Eyepieces}

They enable magnification of images which are produced in binocular tubes. Various range of magnifying quantity is accomplished like " $\times 10, \times 12.5$ and $\times 20$ up to $\times 40$ ". Many restorative and endodontic procedures can be performed with better quality under $\times 10$ magnification. Instrument retrieval or the procedures on tooth apex need a magnifying value of range $\times 15-20$.

A surgical operating microscope has various other attached parts like integrated video graphic devices, units meant to store images, powerful lighting sources, photographic adapters for cameras, device to capture beam splitter, powerful LEDs, color printers \& halide lighting system meant to cause unhindered coaxial illumination.

\section{Lighting Unit}

The principal types of lights used in surgical microscopes are incandescent, halogen \& fiber optic. Halogen lamps have colour temperature that is higher thus producing a whiter light than the lamps which use conventional bulbs. Other choices include xenon lamps that functions ten times longer than halogen lamp. They produce light similar to day light which delivers extraordinarily bright images with sharper contrast.

\section{Mounting System}

Surgical microscopes can be ceiling, wall, and floor mounted. Firstly, the microscopes were fixed and very difficult to operate. Recent microscopes are articulating types that provides easy movement of scope with less strain. Ceiling and wall mounted scopes have fixed place but they help in space management in small working areas. Mounted floor is smoothly transported but a lot of space is occupied.

\section{Steps in the Use of Magnifications}

With increasing application of microscopes, there is a compulsion for each practitioner to learn the technique in stepwise manner to attain total synchronisation in hand \& eye movements during they put this system to use.1,9,12

\section{Working Distance}

It is distance taken from object in vision to eye lens. Also known as working distance. There are many posture regarding problems that clinicians suffer from. Hence depending upon person to person height and length of arms, the WD ranges from $30-45 \mathrm{~cm}$. this range of WD will improve posture and reduce the eye strain.

\section{Working Range (Depth of Field)}

It can be described as the range where object stays within focus. DOF of normal vision has its range from WD to infinity.

\section{Convergence Angle}

Convergence angle is the essential angle which aligns two oculars. This enables them to point in same distance \& this angle may vary with inter pupillary distance (IPD). It states the location of extraocular muscles. There may be tension of internal \& external rectus muscles that cause eye fatigue.

\section{Field of View}

Field of view is the angular extent or the linear size of the object while it is seen using telescopic system.

\section{Interpupillary Distance}

This forms the main adjustment while using any magnification system. Best technique to know our IPD is to focus both the binocular eyepieces. In the beginning 2 images / circles are seen \& they are then adjusted to the point, where both coincide and become one circle. This point is recognised as the IPD and is applied as a permanent reference while using magnifications. There is a different IPD of every individual that are useful in magnification.

\section{Viewing Angle}

Viewing angle describes location of the binocular optics. Its angulations are kept such that a comfortable work position is attained by the operator. A shallower angle requires less tilting of neck for viewing the object.

\section{LOUPES V/S OPERATING MICROSCOPES}

\section{Advantages of Loupes}

They are cost effective. Easier to use initially as they are head mounted. They are less clumsy in the working arena.

\section{Advantages of Operating Microscope}

1. Comfortable to the operator's eye due to the parallel viewing optics, exceptional coaxial fiber optic illumination as well as the varied range of magnification,

2. Innumerable accessories like DVD preparations, photos and video single lens reflex cameras for documenting cases \&, co-observer tubes meant to enable supplementary observation by 3 rd assistant.

3. When microscope is compared to conventional protocol, conventional look like an unrefined crushing \& ripping of tissues.

4. Motor harmonisation is significantly enhanced when meticulous grip instruments are used. This reduces tremble. One of the most useful points of using magnifications is ergonomics. Also, it reduces operator discomfort and fatigue.

Health related issues due to poor ergonomics like disc hernias, spondylosis, impinging of rotator cuff, and neck, back, \& shoulder problems are rising in dental fraternity. Problems associated with muscle balance and their mechanisms are discussed by comprehending the distinction between "posture-directed dentistry" as well as "image-directed dentistry." "Posture directed dentistry" means procedures performed using an indirect line of sight (microscope). "Image directed dentistry" means the procedures that are performed using a direct vision. When viewing an object, the surgical operating microscope allows the dentist to sit erect comfortably with the head, neck, as well as the back arranged in a straight line. While doing a microsurgery, the dentists' seating area is mostly advised as 11 o'clock and 12 o'clock 
positions to avoid twist \& turn movements at the time of procedures.

\section{Applications of Magnification \& Its Effect on Treatment outcome \\ Minimally Invasive Surgical Technique}

In 2007 Cortellini and Tonetti given the concept of minimally invasive surgical technique also called as MIST. This technique was specifically started in isolated infrabony defects for periodontal regeneration, which includes least reflecting of short buccal and lingual flaps having coronoapical and mesiodistal extensions with exposure of coronal part of the residual ridge with defect. In the narrow interdental spaces, papilla preservation flap is given. After refection of flap scaling and root planning is done with the help of mini-curettes \& sonic or ultrasonic tips. Modified minimally invasive technique for surgery, also called as M-MIST includes small interdental access to the defect from buccal side only with application of regenerative material. ${ }^{13-17}$ Clinical success rate of microsurgical approach for periodontal regenerative surgery is verified by multiple case reports and studies. ${ }^{18-20}$

\section{Adhesive Dentistry \\ Ideal restorative margins are key factor for successful and long lasting restorations. Caries close to pulp can be distinguished as infected and affected dentin. This helps to preserve affected dentin \& pulpal exposures. Using the precision under the scope, margin preparation \& outline of crown / veneer preparation can be made perfect.}

\section{endodontics}

Magnification has done great refinement in endodontics which made microscope a very important part of the armamentarium for all endodontic techniques. The capacity to closely view the root canal from both orthograde and retrograde viewpoint has led to the establishment of latest standards for RCT outcomes. Diagnosis of pit \& fissure caries, micro-cracks and fractures, straight line access to apical foramen of tooth, in total deroofing of pulpal chamber, locating of canal orifices (mainly MB2 in the maxillary 1st molars), dentinal map, oval canals, denticles, perforation repairs, fractures, obturation techniques, retrieving of broken files, apical ramifications, retreatment, mainly removing of gutta-percha, and defects or crimps in rotary files, can be traced earlier. This prevent instrument breakage. ${ }^{21-23}$

\section{Errors in the Use of Microscope}

Wrong Magnification Power

Usage of wrong magnification power, i.e., very high or very low will lead to narrower field of vision \& small DOF. Magnifying value that allows operator to work easily without strain and losing focus is ideal. In periodontal surgery, it is up to $\times 5$ for loupes and for microscopes power of $\times 10-20$. Papilla preservation is done under $\times 10-15$, single-tooth root coverage is done under $\times 12-15, \times 6-8$ for clinical inspection / diagnosis, or flap surgery for quadrant, and $\times 15-25$ for endodontics appear to be the ideal range for magnifications. ${ }^{9}$

\section{Inadequate Coordination}

Surgeon \& his helper must have adequate co-ordination for optimal workflow. In micro endodontics, there is less movement of the patient and operator position with straight line vision. Nonetheless, at the time of surgical procedure, there are chances of having a broader field, continuous movement. So, 2 nd helper for ordering armamentarium is necessary,

\section{Lack of Practice or Improper Training}

With proper practice and training operator will develop good hand and eye co-ordination, which will reduce tremors and improve ergonomics. Minimum 6 month learning curve is required.

\section{Recent Advances in Microscopes 24,25,26,27 Zeiss OPMI PROergo}

It has a property of foot-controlled or motorised adjusting of focal length. This lead to least disturbance \& enhanced ergonomic work even in case of treatments continuing for long hours.

\section{Mechanical Optical Rotating Assembly Interface (MORA} Interface)

In this, the binocular tubes are connected at 90 degree to body of surgical operating microscope. Because of this, there is a restricted, independent rotation around the planer axis of the binocular tube. This formulation was done to overcome the shortcomings of conventional microscopes. The older ones had a design which allowed clinicians to be seated at the $9-10$ o'clock position. This resulted in inclination of neck position towards right shoulder. This lead to overextending of left arm, muscle tension, fatiguing, and disability. This technology allows the operating personnel to be positioned at 12 o'clock position, which provide a horizontal WD. This has compatibility with the distance between the head and the mouth of the patient.

\section{Periodontal Endoscope}

It is a newer technique which uses a mini dental endoscope allowing a view of the radicular surface at magnifications of $x$ $24-\times 48$. A $99 \mathrm{~mm}$ fiber-optic bundle which is a mixture of 10,000 pixel capture bundle enclosed by several illumination fibers. This fiber coupled into an explorer is carried to the subgingival margin. The magnified images are displayed on a chairside video screen at once. This allows effective debridement of residual islands of calculus or biofilms.

\section{Varioscope}

Varioscope is a miniature, light weight head mounted dental operating microscope used for surgical navigation. It is also referred to as an Augmented Reality. There is a display of PC generated sceneries. It has an integrated camera for documenting information. The mobility of operator head is the greatest advantage of varioscope. This is contrary to the surgical operating microscopes which lack manoeuvrability because of the cumbersome equipment. 


\section{CONCLUSIONS}

Decreasing operating time and increasing the clinical confidence is a very important part of dentistry. In spite of moderately long learning curve associated with the use of surgical operating microscope, several magnification aids assist in producing high quality dentistry. A strong consideration should therefore be given to adopt this concept. Any equipment that aids in diagnosis and operating procedure is appreciated. However, the success depends upon our commitment to achieve perfection and excellence. If we make a genuine effort, we can find ourselves rejuvenated and the dental practice becomes more enjoyable with the use of magnification.

Financial or other competing interests: None.

Disclosure forms provided by the authors are available with the full text of this article at jemds.com.

\section{REFERENCES}

[1] Tibbetts LS, Shanelec D. Periodontal microsurgery. Dent Clin North Am 1998;42(2):339-59.

[2] Daniel RK. Microsurgery: through the looking glass. New Engl J Med 1979;300(22):1251-7.

[3] Serafin D. Microsurgery: past, present and future. Plast Reconstr Surg 1980;66(5):781-5.

[4] Acland R. Practice manual for microvascular surgery. $2^{\text {nd }}$ edn. St. Louis: C.V. Mosby Publishing Company 1989.

[5] Belcher JM. A perspective on periodontal microsurgery. Int J Periodontics Restorative Dent 2001;21(2):191-6.

[6] Barraquer JI. The history of the microscope in ocular surgery. J Microsurg 1980;1(4):288-99.

[7] Pieptu D, Luchian S. Loupes-only microsurgery. Microsurgery 2003;23(3):181-8.

[8] Friedman M, Mora AF, Schmidt R. Microscope-assisted precision dentistry. Compend Contin Educ Dent 1999;20(8):723-8.

[9] Lang NP, Lindhe J. Periodontal plastic microsurgery. In: Clinical periodontology and implant dentistry. Vol. 2. $6^{\text {th }}$ edn. Oxford, UK: Wiley-Blackwell 2015:1029-44.

[10] Newman MG, Takei HH, Klokkevold PR, et al. Recent advances in surgical technology. In: Carranza's Clinical Periodontology. $10^{\text {th }}$ edn. Philadelphia: W. B. Saunders 2006:1030-8.

[11] Hegde R, Sumanth S, Padhye A. Microscope-enhanced periodontal therapy: a review and report of four cases. J Contemp Dent Pract 2009;10(5):E088-96.

[12] Tibbetts LS, Shanelec DA. An overview of periodontal microsurgery. Curr Opin Periodontol 1994:187-93.
[13] Harrel SK. A minimally invasive surgical approach for periodontal regeneration: surgical technique and observations. J Periodontol 1999;70(12):1547-57.

[14] Cairo F, Carnevale G, Billi M, et al. Fiber retention and papilla preservation technique in the treatment of infrabony defects: a microsurgical approach. Int J Periodontics Restorative Dent 2008;28(3):257-63.

[15] Cortellini P, Tonetti MS. A minimally invasive surgical technique with an enamel matrix derivative in the regenerative treatment of intra-bony defects: a novel approach to limit morbidity. J Clin Periodontol 2007;34(1):87-93.

[16] Cortellini P, Prato GP, Tonetti MS. The modified papilla preservation technique. A new surgical approach for interproximal regenerative procedures. J Periodontol 1995;66(4):261-6.

[17] Cortellini P, Prato GP, Tonetti MS. The simplified papilla preservation flap. A novel surgical approach for the management of soft tissues in regenerative procedures. Int J Periodontics Restorative Dent 1999;19(6):589-99.

[18] Wachtel H, Schenk G, Böhm S, et al. Microsurgical access flap and enamel matrix derivative for the treatment of periodontal intrabony defects: a controlled clinical study. J Clin Periodontol 2003;30(6):496-504.

[19] Kotschy P. Optimal root cleaning and microinvasive periodontal pocket surgery with microscope controlled glass bead blasting. Int J Microdentistry 2010;2(1):48-55.

[20] Francetti L, Fabbro MD, Calace S, et al. Microsurgical treatment of gingival recession: a controlled clinical study. Int $\mathrm{J}$ Periodontics Restorative Dent 2005;25(2):181-8.

[21] Pecora G, Andreana S. Use of dental operating microscope in endodontic surgery. Oral Surg Oral Med Oral Pathol 1993;75(6):751-8.

[22] Kim S, Kratchman S. Modern endodontic surgery concepts and practice: a review. J Endod 2006;32(7):601-23.

[23] Sharma R, Hegde V, Siddharth $M$, et al. endodonticperiodontal microsurgery for combined endodonticperiodontal lesions: an overview. J Conserv Dent 2014;17(6):510-6.

[24] Mukherjee P, Patel A, Chandak M, et al. Minimally invasive endodontics a promising future concept: a review article. Int J Sci Stud 2017;5(1):245-51.

[25] Hande A, Lohe V, Chaudhary M, et al. Impact of virtual microscopy with conventional microscopy on student learning in dental histology. Dent Res J (Isfahan) 2017;14(2):111-6.

[26] Tandon S, Venkiteswaran A, Baliga SM, et al. Recent research trends in dentistry. J Indian Soc Pedod Prev Dent 2017;35(2):102-5.

[27] Doornbusch H, Veenstra K. Frequently asked questions about the dental microscope. The Microscope in Dentistry. Editorial. Germany: Carl Zeiss Surgical GmbH Company 2007. 